\title{
Mechanisms of the Antiangiogenic Effects for Cancer by the Aspirin
}

\author{
Bin Yang ${ }^{1}$, Shiyuan Xie ${ }^{1}$, youqiong wang ${ }^{1}$, and Yixuan Huang ${ }^{1}$ \\ ${ }^{1}$ Guangxi Medical University
}

May 18, 2020

\begin{abstract}
Aspirin as an old drug extracted from willow bark and widely used in the prevention and treatment of cardiovascular diseases. Increasing evidence have shown that aspirin use may significantly reduce the angiogenesis of cancer, while the mechanism of the association between angiogenesis and aspirin is complex. Although COX-1 is widely known as a target of aspirin, several studies reveal other antiangiogenic targets of aspirin, such as angiotensin II, Glucose transporter 1, heparanase, and matrix metalloproteinase. In addition, some evidence indicates that aspirin may produce antiangiogenic effects after acting in different cell types, such as endothelial cell, platelet, pericyte, and macrophage. In this review, we concentrate on recent researches on the antiangiogenic effects of aspirin in cancer, and analyze the molecular mechanisms of aspirin and its metabolites. Moreover, we discuss some mechanisms through which aspirin treatment may normalise existing blood vessels, including preventing disintegration of endothelial adheren junctions and recruiting pericytes. We also address the antiangiogenic effects and the underlying mechanisms of aspirin derivatives, which aim to improve safety and efficacy.
\end{abstract}

\section{Introduction}

Angiogenesis is the process through which new capillaries are sprouted from pre-existing ones (Eilken et al., 2010). And the components of the vascular wall are basement membrane, pericytes, vascular smooth muscle cells and endothelial cells (Carmeliet et al., 2011). The concept of antiangiogenic therapy, inhibition of angiogenesis could inhibit tumor growth, started after an observation by Folkman discovering new vessel production during tumors growth (Folkman, 1971). It is now relatively cleared that angiogenesis plays an important role in tumor metastasis and growth (Vasudev et al., 2014; Bielenberg et al., 2015). The angiogenic system is required for tumor tissue to grow and develop, and angiogenesis is a complex process involving an interaction between soluble factors, extracellular matrix (ECM) components and endothelial cells(ECs) (Patan, 2000). The early stage of angiogenesis occurs dynamic functions of ECs including proliferation, migration and maturation (Griffioen et al., 2000). The significant event in tumor angiogenesis is endothelial cell migration, which relys on the assembly of protease-protein complexes in front of migrating cells to degrade the basement membrane and invade the matrix of adjacent tissues (Ausprunk et al., 1977; Liekens et al., 2001). Notably, the chronic imbalance of proangiogenic and antiangiogenic factors leads to abnormal angiogenesis in tumor tissues (Goel et al., 2011; Carmeliet et al., 2011).

Aspirin (acetylsalicylic acid, ASA) is an old medicine that was originally extracted from willow bark and is widely used in the prevention and treatment of cardiovascular and cerebrovascular diseases (Desborough et al., 2017). It is absorbed in the gastrointestinal tract after oral administration of aspirin in man, and then converted into salicylic acid (SA) which is further metabolized to salicyhxic acid, gentisic acid, gentisuric acid (2,5-DHBA), salicyl acyl glucuronide, and salicyl phenolic glucuronide (Rumble et al., 1981). During the circulation process, the biological activity of salicylic acid is impaired due to the large amount of salicylic acid (50-80\%) bound to albumin, which is because the free salicylic acid has biological activity (Hua et al., 2019).

Increasing evidence have shown that long-term regular aspirin use can significantly reduce the risk of overall 
cancer (Cao et al., 2016). These effects may be related to the antiangiogenic ability of aspirin (Pearce et al., 2003; Yoshida et al., 2003; Tsujii et al., 1998). Here, we update recent researches on the antiangiogenic effects of aspirin in cancer prevention and treatment, and analyze the several molecular mechanisms of the antiangiogenesis effects for cancer by aspirin.

\section{Antiangiogenic mechanisms for the prevention and therapy of cancer by aspirin}

In angiogenesis, the therapeutic concentration of aspirin can cause a significantly reduction in vessel tubule formation, while the high levels of endothelial cell apoptosis could triggered by aspirin at the higher concertration (Borthwick et al., 2009). Moreover, present studies show that aspirin significantly inhibits proliferation of normal human dermal microvascular endothelial cells (HuDMEC) and human umbilical vein endothelial cells (HUVEC) (Pearce et al., 2003; Boonmasawai et al., 2009). Furthermore, ASA also blocks endothelial cell migration after stimulation with VEGF (Navone et al., 2018), cancer cell (Maity et al., 2019) or ADP (Battinelli et al., 2011). However, the mechanism of these effects is complex. Many endogenous angiogenesis stimulators and inhibitors reside within the host microenvironment, including Interleukin-8 (IL-8), Fibroblast growth factor-1 (acidic FGF, FGF1), Fibroblast growth factor-2 (basic FGF, FGF2), Vascular endothelial growth factor (VEGF), vascular permeability factor (VPF) and Platelet-derived growth factor (PDGF) as well as others (Li et al., 2012). Aspirin could suppress angiogenesis and curb tumor growth by inducing these inhibitors and inhibiting these stimulants (Figure 1).

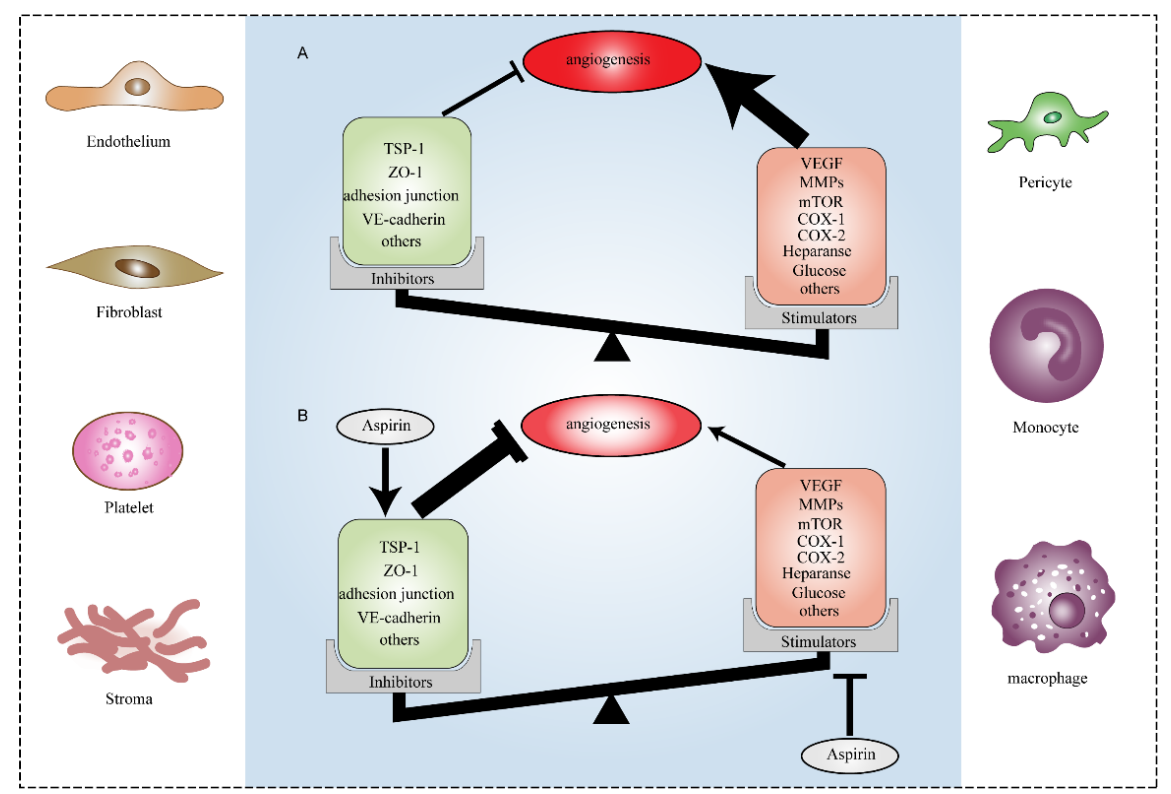

Figure 1 The role of aspirin to tumor angiogenesis inhibition by regulation a balance of endogenous inhibitors andstimulators. (A) In the tumor microenvironment, the sum effect of these inhibitors and stimulants is angiogenesis with abnormal structure and function. This imbalance proangiogenic effects is aggravated by activated platelets, monocytes and macrophages. (B) Aspirin may reverse the balance of antiangiogenic and proangiogenic effect in angiogenesis through lower endogenous stimulators and boost inhibitors. Reverse of antiangiogenic and proangiogenic balance may inhibit angiogenesis, normalize existing blood vessels and reduce permeability and leakiness.

Abbreviations: TSP-1, thrombospondin-1; ZO-1, Zonula Occludens-1; VEGF, Vascular endothelial growth factor; FGF, Fibroblast growth factor; MMPs, matrix metalloproteinases; mTOR, Mammalian target of rapamycin.

\subsection{Effect on Heparanase and the matrix metalloproteinases}


Heparanase,the heparan sulfate (HS)-degrading endo- $\beta$-D-glucuronidase,could regulate angiogenesis and degrade the HS chain of heparan sulfate proteoglycans in the extracellular matrix (Li et al., 2009; Vlodavsky et al., 2018). After the extracellular matrix collapses, heparin-binding cytokines such as HGF, VEGF, and bFGF are released to promote tumor angiogenesis (Talmadge et al., 2010; Vlodavsky et al., 2001). Notably, except for the members of FGF-19 subfamily with little or no affinity for these glycosaminoglycans,all fibroblast growth factors high affinitive bind to heparin (Asada et al., 2009). Heparanase is inhibited by aspirin which directly bind to Glu150 (human Q9Y251: Glu 225) region and thereby suppress the vascular structures formation in a dose-dependent manner (Dai et al., 2017). Salicylic acid also has the same effect of inhibiting the heparanase activity (Dai et al., 2017). Therefore, aspirin or salicylic acid may reduce release of heparin-binding cytokines such as HGF, VEGF, and bFGF, thereby inhibiting angiogenesis.

The matrix metalloproteinases (MMPs), a large family of zinc-dependent endoproteases, can remodel and degrade the components in the extracellular matrix (ECM) and could play a vital role in angiogenesis (Kapoor et al., 2016). The degradation effect of Gelatinase-B (MMP-9) in the extracellular matrix promotes the migration of ECs to angiogenesis stimuli (Hiraoka et al., 1998; Puyraimond et al., 1999). Moreover, MMP-9 can degrade the Multimerin 2, which is tightly juxtaposed with ECs surface and bind proangiogenic cytokines to exert angiostatic functions (Andreuzzi et al., 2017; Christian et al., 2001). Metalloproteinase 1 (TIMP-1) as a specific tissue inhibitor could inhibit MMP-9 and suppress the ECM degradation in tumor angiogenesis (Khokha et al., 1994). In addition, it has been demonstrated that MMP-9 can induce the expression of VEGF (Bergers et al., 2000). Interesting, aspirin can suppress the levels of secreted MMP-9 activity and increase the levels of secreted TIMP-1 activity in the cancer cell (Shi et al., 2017). In this way, aspirin may inhibit migration of ECs to angiogenesis stimuli in the tumor microenvironment. Furthermore, EP3 receptor signal transduction on ECs is crucial for MMP-9 upregulation, which can enhance tumor angiogenesis (Amano et al., 2008). It has been demonstrated that aspirin can reduce MMP-9 upregulation by inhibiting cPLA2-COX-1-PGE2-EP3 Signaling in ECs (Salvado et al., 2013).

\subsection{Effect on Cyclooxygenase of endothelial cells}

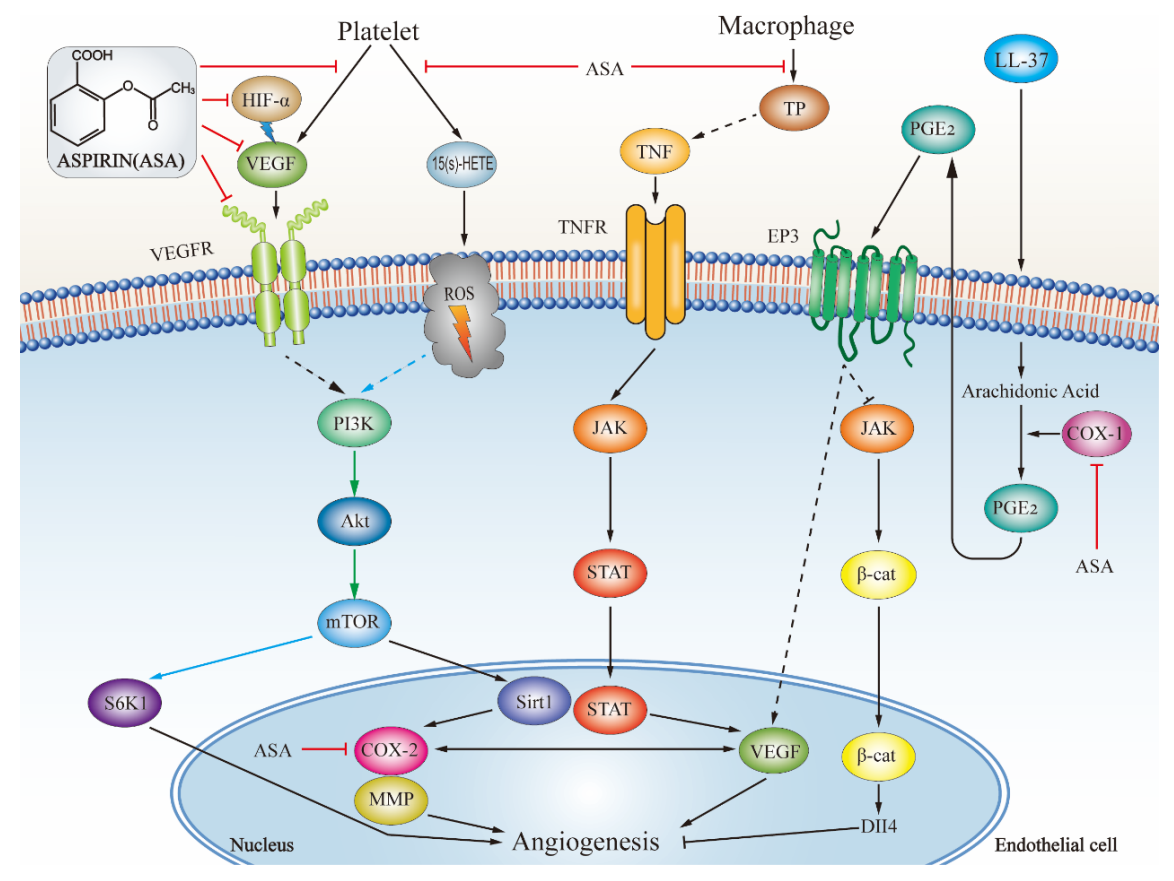

The expression and activity of COX-1 in HUVEC regulates the formation of tubular structures induced by cancer cells (Tsujii et al., 1998). LL-37, the only cathelicidin protein expressed by humans, upregulated in 
breast, ovarian and lung tumors (Larrick et al., 1995; Coffelt et al., 2009; Weber et al., 2009; Haussen et al., 2008).The Cathelicidin LL-37 can induce a proangiogenic response via cPLA2-COX-1-PGE2-EP3 Signaling in human ECs, which is suppressed by aspirin in a manner of inhibiting COX-1 in vivo (Salvado et al., 2013) (Figure 2). Activation of PGE2/EP3 axis promotes sprouting angiogenesis by suppression of protein kinase A (PKA)/p-S675- $\beta$-catenin/Notch signaling in ECs (Chen et al., 2017). Moreover, fibroblast growth factor-1 and fibroblast growth factor- 2 are the first two discovered polypeptides showing proangiogenic effects (Thomas et al., 1985; Esch et al., 1985). FGFR1 (Fibroblast Growth Factor Receptor 1) pathway in microvascular endothelial cells can be activated by EP3 receptor signal mediation (Federica et al., 2008). The 2,5-dihydroxyphenylic acids (Gentisic Acid), a metabolite of Aspirin, is a powerful fibroblast growth factor Inhibitor (Grootveld et al., 1998; Fernández et al., 2010). Furthmore, angiogenic effects of PGE2 are mediated by up-regulation of the C-X-C chemokine receptor type 4 (CXCR4) and CXCR4 expression can be inhibited by aspirin in HMECs (Rosalba et al., 2003). However, it warrants further research to verify the downstream of cPLA2-COX-1-PGE2-EP3 signalling transduction when aspirin antiangiogenesis.

Figure 2 Shown some signalling pathways that are targets of aspirin to inhibit tumor angiogenesis.However, it warrants further study to verify the downstream of signalling transduction in these pathways.

It has been demonstrated that COX-2 in ECs is vital in regulating angiogenesis, and the microvessel density relate to the level of COX-2 expression (Jones et al., 1999; Kazuhiko et al., 2000). Moreover, in the sponge implanted mouse model, COX-2 mediated prostaglandins can up-regulate the expression of VEGF mRNA (Yoshida et al., 2003).

However, aspirin effectively inhibits COX-1 activity at a pharmacological concentration, but has a weaker active to block COX-2 activity (Meade et al., 1993; Mitchell et al., 1993). In fact, it has been demonstrated that salicylate inhibits Cyclooxygenase- 2 transcription induced by IL- $1 \beta$ or phorbol 12 -myristate 13 -acetate by blocking $\mathrm{C} /$ Enhancer-binding Protein $\beta$ binding to its cognate site on Cyclooxygenase-2 promoter (Michael et al., 2001). Furthermore, aspirin and sodium salicylate at pharmacological concertration blocked COX-2 protein expresion induced by cancer and VEGF (Shtivelband et al., 2003) (Figure 2). COX-2 protein levels in HUVECs, inducing by PMA (Porbolol 12-myristate 13-acetate), inhibited by Aspirin and sodium salicylate in the manner of concentration-dependent (Xu et al., 1999). Therefore, aspirin may inhibit COX-1 activity and COX-2 transcription to regulate angiogenesis.

\subsection{Effect on platelet-mediated angiogenesis}

Platelets are considered to be the main actors in each step of angiogenesis as they store various growth factors, proteases, cytokines, chemokines and cell adhesion molecules (Patzelt et al., 2012; Pipili-Synetos et al., 1998). Therefore, pharmacological interventions of platelet-associated proangiogenic activity have been recognized as a important adjuvant treatment for cancer (Bambace et al., 2011; Radziwon-Balicka et al., 2012).

The 15(S)-hydroxyeicosatetraenoic acid (15(S)-HETE), a direct COX-1 product in platelet, can combine with other pro-angiogenic factors released by platelets to regulate angiogenesis (Rauzi et al., 2016). Aspirin can block the angiogenic response of thrombin-stimulated human platelets in human microvascular endothelial cells (HMEC-1) (Etulain et al., 2013). In addition, aspirin inhibits 15(S)-HETE production in platelet with inhibition COX-1 to reduce angiogenesis, which also provide a explanation for the protective effects of lowdose aspirin on some cancers (Rauzi et al., 2016) (Figure 2). A study demonstrates that 15(S)-HETE can promote angiogenesis in HuDMECs through the PI3K-Akt-mTOR-S6K1 signaling pathway (Zhang et al., 2005). However, it warrants further study to verify the downstream of 15(S)-HETE signalling transduction when aspirin antiangiogenesis.

Platelet levels of thrombospondin-1 (TSP-1) have been indicated to regulate early stage of tumor angiogenesis (Zaslavsky et al., 2010). In breast cancer patients, taking aspirin therapy can upregulate TSP-1 level without a concurrent increase in VEGF levels in platelets (Holmes et al., 2013). It is also indicated that pretreatment with aspirin could reduce the amount of VEGF released from platelets at rest and after being 
activated by ADP or interacting with MCF-7 cells (Battinelli et al., 2011). Furthermore, aspirin may correct abnormal platelet activation, prevent the release of a large number of angiogenesis regulators, and then help normalize the tumor vasculature and shape the tumor microenvironment, thereby reducing tumor invasion and progression (Su et al., 2014). There is a registered clinical trial to test the efficacy of aspirin use for exploring the platelet function of its mechanism of action, such as the change levels of COX-1, thrombospondin 1, NF-Kb and VEGF (Table1).

\subsection{Effect on Hypoxia stimulation and GLUT1 expression}

Neoplasms have been usually described as hypoxic structures, inefficient of oxygen transportation, and bearing twisted irregular vascular networks (Vaupel, 2004). Hypoxia-reoxygenation (HR), a major driver for tumor angiogenesis, stimulates proliferation of ECs via several signaling pathways including VEGF and transforming growth factor (TGF $\beta 1$ ) (Khaidakov et al., 2011; Hu et al., 2008). Moreover, overexpression of TGF $\beta$-R1 shows angiostatic effect, while blocking TGF $\beta$-R1 can facilitate VEGF-mediated capillary formation and activation of genes related to angiogenesis (Liu et al., 2009). Aspirin effectively inhibits the angiogenic response involved in HR-mediated TGF $\beta 1$ mRNA transcription and TGF $\beta 1$-R1 upregulating, thereby inhibiting HR-stimulated tube formation in HUVECs (Khaidakov et al., 2011). In addition, aspirin can inhibit LOX-1-NADPH oxidase pathway in HUVECs to suppress HR-induced tube formation (Khaidakov et al., 2010).

Glucose transporter 1 (GLUT1) is the main route to uptake glucose in ECs (Goveia et al., 2014). The expression of GLUT1 is promoted in ECs by hypoxia, and the high expression of GLUT1 is related to neoplasm angiogenesis (Zapata-Morales et al., 2014; Semaan et al., 2011). In the process of switching from a quiescent state to angiogenic phenotype, glycolysis provides the essential energy and promotes sprouting of blood vessels during tumor-induced angiogenesis (Rivera et al., 2014). Interesting, aspirin can not only inhibit the expression level of GLUT1 mRNA and protein resulting in impaired glucose uptake ability, but also inhibit the intracellular lactate synthesis and ATP of SEND cells (rat vascular endothelial cell line) (Hu et al., 2014 ). Moreover,taking high-dose aspirin, even reaching $90 \mathrm{mM}$, does not produce side effects that interfere with glycolysis and glucose uptake in human erythrocytes (Worathumrong et al., 1975). Thus, aspirin inhibits the sprouting of blood vessels through regulating the expression level of GLUT1 and inhibiting lactate synthesis. However, it warrants further study to clarify the detailed mechanisms of aspirin in these phenomenon.

\subsection{Effect onrenin-angiotensin system}

The renin-angiotensin system (RAS) is involved in a coordinated hormone cascade that regulates fluidelectrolyte balance and arterial pressure by controlling cardiovascular, adrenal and renal functions (Peach et al., 1997). Some studies suggest that angiotensin II (Ang II) in low concentrations can induce angiogenesis by activating angiotensin type 1 receptor (AT1R) and nicotinamide-adenine dinucleotide phosphate (NADPH) oxidase (Skultetyova et al., 2007; Ushio-Fukai et al., 2006; Hu et al., 2007). MAP kinase activation is the downstream of NADPH oxidase, and inhibition of p38 and p44/42 MAP kinase can reduce angiogenesis (Hu et al., 2007; Seeger et al., 2010). Moreover, it has been noted that suppression of bradykinin degradation and angiotensin II(Ang II) synthesis in RAS can produce a net antiangiogenic state in tumorgenesis (Heffelfinger et al., 2007). Importantly, ASA and SA significantly reduce Ang II-induced expression of AT1R, VEGF, NADPH oxidase subtypes, p-p38 and p-p44/42 MAPKs in endothelial cells, and inhibite angiogenic effects of Ang II in capillary formation (Mitra et al., 2012). It warrants further study to research the effect of aspirin in renin-angiotensin system in clinical setting.

\subsection{Effect on mononuclear phagocytic system}

Within tumor microenvironment, cancer cells induce monocytes to differentiate for tumor-associated macrophages (TAMs) through recruiting monocytes by secreting chemotactic factors such as VEGF, macrophage chemoattractant protein-1 (MCP-1) and macrophage colony-stimulating factor (Solinas et al., 2009). Aspirin could disrupt the crosstalk of angiogenic cytokines between macrophages and $4 \mathrm{~T} 1$ breast cancer cells (Hsieh et al., 2018). Macrophages, including categorization of M1 and M2 phenotype, have the 
potentiality to convert the M1 into the M2 macrophages (Gordon et al., 2010; Spiller et al., 2015). M1 macrophages secrete factors that are involved in initiating the stages of angiogenesis, while M2 macrophages secrete factors are responsible for later process of angiogenesis (Spiller et al., 2014). Importantly, it has been shown that aspirin treatment raised the level of M1 marker expression, but decreased expression of M2 marker (Hsieh et al., 2018).

Thymidine phosphorylase (TP) is a proangiogenic factor that found to be chemotactic for ECs and to induce angiogenesis in vivo (Finnis et al., 1993; Miyadera et al., 1995). TP is highly expressed in macrophages of normal human tissues (Fox et al., 1995). Comparing with adjacent uninvolved tissues, TP is often overexpressed in solid tumors, and its expression is associated with higher microvessel count, increased tumor invasiveness and metastasis (Takebayashi et al., 1996). It also found that TP-expressing cancer cells secreted angiogenic factors (IL-8, bFGF and TNF $\alpha$ ) that stimulated endothelial cells migration and invasion (Elamin et al., 2015). Moreover, activated macrophages are one of the main sources for Tumor Necrosis Factor- $\alpha$ $(\mathrm{TNF} \alpha)$, and it has been suggested that the proangiogenic activity of macrophages is mainly mediated by TNF $\alpha$ (Beutler et al., 1986; Leibovich et al., 1987). Aspirin inhibits MCP-1 and interleukin-8 expression in TNF-alpha stimulated HUVECs (Yang et al., 2004). Besides, THP-1 monocytes induce TP expression by autocrine TNF $\alpha$, and aspirin inhibits TP expression in THP-1 cells by reducing NF $\varkappa$ B DNA-binding activity in the pathway of TNF $\alpha$-induced TP expression (GENG et al., 2003). However, it warrants further studies to determine the downstream of TP signalling transduction in ECs when aspirin antiangiogenesis.

\subsection{Effect on intercellular junctions andPericytes}

Disorganization of tumor vascular layers leads to increased permeability and leakiness, which is a common feature of tumor angiogenesis (Weis et al., 2011). The low vascularity, abnormal morphology, and high permeability of the tumor vessels leads to inefficient delivery of drugs and oxygen into tumors (Jain et al., 1998). Antiangiogenic drugs would be expected to inhibit the angiogenesis and may stop the growth of tumor tissues but not necessarily influence existing blood vessels or cause tumor shrinkage (Baluk et al., 2005).

VEGF, as a pro-angiogenic factor, not only plays a key role in inducing endothelial cell migration, proliferation and differentiation, but also can destroy tight junction proteins, thereby increasing vascular permeability and triggering tumor metastasis (Cross et al. 2013; Azzi et al., 2013). ASA significantly reduced the expression of VEGF in HUVEC,hTNFered the VEGF release from endothelial cells of human primary glioblastoma and blocked the VEGF-promoted angiogenesis (Maity et al., 2019; Navone et al., 2018). Besides, salicylate decreased serum VEGF levels and reduced microscopic vascular structures in animal models (Ghezzo et al., 2004).

Moreover, three types of junctions in the endothelial monolayer have been described: adherens, tight and gap junctions (Bazzoni et al., 2004). The destruction of the adhesion junctions is a vital step in inducing angiogenesis and a prerequisite for releasing EC from the contact inhibition exerted by the association of VE-cadherin and $\beta$-catenin (Khaidakov et al., 2010). Immunocytochemistry performing to p120 catenin and VE-cadherin showed that adherens junctions in VEGF-induced HUVECs tube formation are severe disrupted, but the disruption effects is largely prevented by aspirin and salicylic acid with preincubated in cultures (Khaidakov et al., 2012). Furthermoer, aspirin rescues vascular permeability and up-regulates the expression of VE-cadherin and Zonula Occludens-1 (ZO-1) in MDA-MB-231 conditioned medium (Maity et al., 2019). Aspirin also prevents disintegration of endothelial adheren junctions caused by Hypoxiareoxygenation (HR) (Khaidakov et al., 2010 ).

Pericytes play a vital role in stabilization of blood vessel architecture and regulate vessel permeability (Bergers and Song 2005; Ribeiro et al., 2015). It has been suggested that aspirin can recruit pericytes from multipotent stem cells and help in binding with ECs, which is a sign of normalization of blood vessels and reduces permeability and leakiness through endothelial cell layer (Maity et al., 2018). Thus, normalized tumor vasculature may reinforce drug delivery to further solid tumors.

\section{Antiangiogenic effects of ASA combination with actual proven therapies}


The abnormal tumor vessels leads to inefficient delivery of drugs and oxygen into tumors (Jain et al., 1998). Thus, the normalization of the tumor vasculature by antiangiogenic drugs may reinforce other drugs delivery to further solid tumors, thereby producing an enhancement effect. As targeting angiogenesis is rising a great promise in patients as an antitumor therapy in combination with existing chemotherapies (Ma et al., 2008), we analyze antiangiogenesis properties of coadministration with aspirin.

The previous study has revealed that following coadministration with aspirin therapy, VEGF levels decreased in women with breast cancer relative to levels of receiving Tamoxifen therapy alone (Holmes et al.,2013). Combination therapy of ASA drugs with a VEGF inhibitor block angiogenesis more effectively than either agent alone (Huang et al., 2016). In addition, the administration of aspirin as an adjuvant in combination with Temozolomide, Bevacizumab and Sunitinib showed a synergistic effect on reduce capillary-like tube length in comparison to each exposure alone, in fact aspirin enhanced Temozolomide, Sunitinib and Bevacizumab effect, decreasing significantly Glioblastoma-endothelial cells VEGF-stimulated angiogenesis, especially at the high concentration (Navone et al., 2018). Also, the expression of VEGFR-2, HIF-1 $\alpha$, anti-apoptotic BCL-2 and PI3K/AKT suffered a reduction after combined therapy of Temozolomide, Bevacizumab, Sunitinib and aspirin compared to single-drug treatment (Navone et al., 2018).

5-Fluorouracil (5-FU), a rationally synthesized antitumor agent, is widely used to treat several common malignant tumors, including cancer of the skin, colon and breast (Diasio et al., 1989). The expression of VEGF-A and VEGF-C is significantly reduced in the tumors treated with 5-FU combination with aspirin, and the antitumor effect of 5-FU is synergistically enhanced by aspirin (Zhang et al., 2013). There are three registered clinical trials involved in evaluate the antiangiogenesis efficacy of coadministation aspirin in treating cancers (Table1).

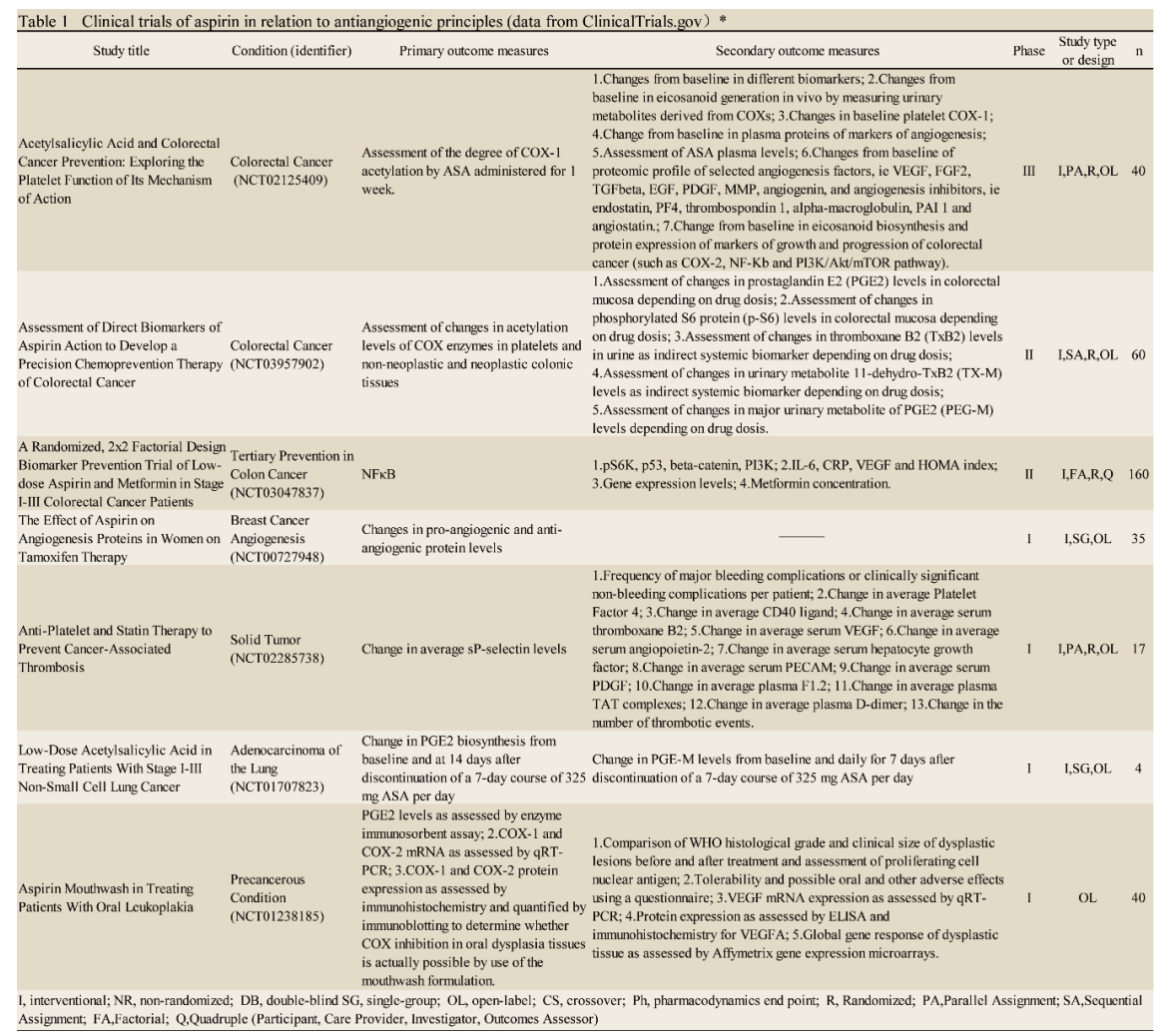

\section{Antiangiogenic effects onnovel derivatives of aspirin and ATL-1}

Some less serious adverse events of aspirin are involved in stomach pain, vomiting, nausea and heart-burn 
(Hua et al., 2019). Long-term aspirin therapy may induce gastrointestinal injury. Even low-dose aspirin can damage the gastroduodenal mucosa in varying degrees, including ulcers, erosions, and bleeding (Lavie et al., 2017). Another adverse event of aspirin is aggravating respiratory diseases such as asthma and chronic sinusitis with nasosinusal polyposis (Pavón-Romero et al., 2017). Thus, in order to become greater safety and greater efficacy compared with ASA, the new derivatives of aspirin are needed.

The NO-donating non-steroidal anti-inflammatory drugs are expected to bring these two promising features for cancer prevention and treatment relative to traditional similar drugs (Yeh et al., 2004; Rigas et al., 2007). For NO-releasing aspirin, NO itself could induce COX2 expression (Basudhar et al., 2017), but aspirin may inhibit it thereby neutralizing the harmful effect of NO. In that way, it is expected that novel derivative of NO-releasing aspirin might show less gastrointestinal injury than aspirin. It has been demonstrated that NOdonating aspirin supresses tumor angiogenesis by inhibiting VEGF expression and reducing the microvessel density in cancer mice xenografts (Ouyang et al., 2008).

PGE2 as a downstream mediator play a major role in regulating angiogenesis, and suppression of its formation is involve in antiangiogenic effects (Méric et al., 2006). Co-ASS, $\mathrm{Co}_{2}(\mathrm{CO})_{6}$ alkyne derivatives of aspirin (Coaspirin), significantly inhibited cellular $\mathrm{PGE}_{2}$ formation from arachidonic acid in breast tumor cells (Ingo et al., 2009). Besides, the zebrafish embryos, an established model organism for studying angiogenesis and vascular development, treat with Co-ASS showed severe defects in angiogenesis, which is manifested in the lack or impaired formation of dorsal longitudinal anastomotic vessels and intersegmental vessels, as well as the reduction of intestinal veins (Lawson et al., 2002; Ott et al., 2009).

Moreover, aspirin-PC is another derivative of aspirin in which PC-enrichedsoylecithin is formulated with aspirin. In short-term clinical trials, the development of PC-related aspirin has been proved to be less harmful to the gastroduodenal mucosa because of its ability to protect the hydrophobic barrier property of the tissue surface (Cryer et al., 2011). Previous study demonstrate that treatment with aspirin-PC significantly reduce the level of VEGF and the number of tumor microvessels in ovarian tumor tissue, especially when aspirin-PC is used in combination with bevacizumab (Huang et al., 2016).

It has been shown that, when COX-2 acetylated by aspirin, the ability of generating prostanoids is blocked, yet acetylized COX-2 remains active in epithelial cells, mononuclear cells and endothelial cells and initiates the biosynthesis of new product of transcellular biosynthesis or cell-cell interactions termed as aspirintriggered-15-epi-lipoxins (ATLs) (Clària et al., 1995). The previously studies reveal that 15-epi-16-(parafluoro)-phenoxy-lipoxin A4 (ATL-1), an ATLs stable analog, inhibited VEGF-stimulated endothelial cell proliferation and migration in a concentration-dependent manner (Fierro et al., 2002). Moreover, VEGFdriven HUVEC migration requires the synergistic activation of two complementary pathways: one pathway involves the activation of SAPK2/p38 MAP kinase-mediated actin, and the other involves the phosphorylation of focal adhesion kinase (FAK) and the assembly of focal adhesions (Rousseau et al., 2000). Treatment of HUVEC with ATL-1 significantly reduced VEGF-induced p38 and FAK phosphorylation (Cezar-de-Mello et al., 2006). Besides, the multiple steps in the angiogenesis process also inhibited by ATL-1, including VEGF-induced EC proliferation, MMP-9 activity, HUVEC adhesion and VEGFR-2 phosphorylation (Cezarde-Mello et al., 2008).

\section{Conclusion}

Based on these knowledge, aspirin is an intriguing and promising antiangiogenesis agent. We have addressed here the some potential antiangiogenic effects of aspirin therapy in not only decreasing endothelial cell proliferation and migration, but also in reducing macrophage or platelet-mediated angiogenesis. We have also discussed some mechanisms through which aspirin treatment may normalise existing blood vessels, including preventing disintegration of endothelial adheren junctions and recruiting pericytes, thereby reducing permeability and leakiness through endothelial cell layer. When a single-molecule is blocked, the tumor may bypass this angiogenic protein through overexpression of other angiogenic factors, resulting in a low therapeutic effect. The above drawback may be overcome by aspirin inhibiting several angiogenic molecules simultaneously, such as VEGF, cyclooxygenase, matrix metalloproteinase, and heparanase. Furthermore, 
more clinical trials are needed to validate the antiangiogenic mechanisms and benefits of aspirin therapy, especially in combination with existing chemotherapies to prevent various human tumors, such as glioblastoma, breast cancer and colorectal cancer. However, angiogenesis is essential for reproductive function and wound healing, so the dose of aspirin should be quantified to inhibit tumor angiogenesis, but it does not injure healthy endothelial function and maintenance. The novel derivatives could be designed from aspirin backbone to be more antiangiogenic and more suitable as an antiangiogenesis agent while overcoming the side effects of aspirin.

\section{Conflict of interest statement}

The authors declare no conflict of interest.

\section{References}

Amano H, Ito Y, Suzuki T, Kato S, Matsui Y, Ogawa F, et al. (2008). Roles of a prostaglandin E-type receptor, EP3, in upregulation of matrix metalloproteinase-9 and vascular endothelial growth factor during enhancement of tumor metastasis. Cancer Science 100(12): 2318-2324.

Andreuzzi E, Colladel R, Pellicani R, Tarticchio G, Cannizzaro R, Spessotto P, et al. (2017). The angiostatic molecule Multimerin 2 is processed by MMP-9 to allow sprouting angiogenesis. Matrix Biology 64: 40-53.

Asada M, Shinomiya M, Suzuki M, Honda E, Sugimoto R, Ikekita M, et al. (2009). Glycosaminoglycan affinity of the complete fibroblast growth factor family. Biochimica et Biophysica Acta 1709(1): 40-48.

Ausprunk DH, Folkman J (1977). Migration and proliferation of endothelial cells in preformed and newly formed blood vessels during tumor angiogenesis. Microvascular research 14: 53-65.

Azzi S, Hebda JK, Gavard J (2013). Vascular permeability and drug delivery in cancers. Frontiers in oncology 3: 211 .

Baluk P, Hashizume H, McDonald DM (2005). Cellular abnormalities of blood vessels as targets in cancer. Current Opinion in Genetics \& Development 15: 102-11.

Bambace NM 1, Holmes CE (2011). The platelet contribution to cancer progression. Journal of Thrombosis and Haemostasis 9: 237-249.

Basudhar D, Glynn SA, Greer M, Somasundaram V, No JH, Scheiblin DA, et al. (2017). Coexpression of NOS2 and COX2 accelerates tumor growth and reduces survival in estrogen receptor-negative breast cancer. Proceedings of the National Academy of Sciences of the United States of America114(49).

Battinelli EM, Markens BA, Italiano JE Jr (2011). Release of angiogenesis regulatory proteins from platelet alpha granules: modulation of physiologic and pathologic angiogenesis. Blood 118(5): 1359-1369.

Bazzoni G, Dejana E (2004). Endothelial cell-to-cell junctions: molecular organization and role in vascular homeostasis. Physiological reviews 84: 869-901.

Bergers G, Brekken R, McMahon G, Vu TH, Itoh T, Tamaki K, et al. (2000). Matrix metalloproteinase-9 triggers the angiogenic switch during carcinogenesis. Nature Cell Biology 2 (10): 737-744.

Bergers G, Song S (2005). The role of pericytes in blood-vessel formation and maintenance. Neuro-Oncology 7: 452-464.

Beutler B, Cerami A (1986). Cachectin and tumour necrosis factor as two sides of the same biological coin. Nature 320(17): 584-588.

Bielenberg DR, Zetter BR (2015). The Contribution of angiogenesis to the process of metastasis. Cancer journal (Sudbury, Mass.) 21: 267-273.

Boonmasawai S, Akarasereenont P, Techatraisak K, Thaworn A, Chotewuttakorn S, Palo T (2009). Effects of selective COX-inhibitors and classical NSAIDs on endothelial cell proliferation and migration induced by 
human cholangiocarcinoma cell culture. J Med Assoc Thai 92 (11): 1508-1515.

Borthwick GM, Johnson AS, Partington M, Burn J, Wilson R, Arthur HM (2009). Therapeutic levels of aspirin and salicylate directly inhibit a model of angiogenesis through a Cox-independent mechanism. The FASEB Journal 20(12): 2009-16.

Cao Y, Nishihara R, Wu K, Wang M, Ogino S, Willett WC, et al. (2016). Population-wide impact of longterm use of aspirin and the risk for cancer. JAMA Oncology 2(6): 762-9.

Carmeliet P, Jain PK (2011). Principles and mechanisms of vessel normalization for cancer and other angiogenic diseases. Nature Reviews Drug Discovery 10(6): 417-27.

Carmeliet P, Jain RK (2011). Principles and mechanisms of vessel normalization for cancer and other angiogenic diseases. Nature Reviews Drug Discovery10(6): 417-427.

Cezar-de-Mello PF, Nascimento-Silva V, Villela CG, Fierro IM. (2006). Aspirin-triggered Lipoxin A4 inhibition of VEGF-induced endothelial cell migration involves actin polymerization and focal adhesion assembly. Oncogene 25(1): 122-129.

Cezar-de-Mello PF, Vieira AM, Nascimento-Silva V, Villela CG, Barja-Fidalgo C, Fierro IM (2008). ATL-1, an analogue of aspirin-triggered lipoxin A 4, is a potent inhibitor of several steps in angiogenesis induced by vascular endothelial growth factor. British Journal of Pharmacology 153(5): 956-965.

Chen D, Tang J, Wan Q, Zhang J, Wang K, Shen Y, et al. (2017). E-prostanoid 3 receptor mediates sprouting angiogenesis through suppression of the protein kinase $\mathrm{A} / \beta$-catenin/notch pathway. Arteriosclerosis, Thrombosis, and Vascular Biology 37: 856-866.

Christian S, Ahorn H, Novatchkova M, Garin-Chesa P, Park JE, Weber G, et al. (2001). Molecular cloning and characterization of EndoGlyx-1, an EMILIN-like multisubunit glycoprotein of vascular endothelium. The Journal of biological chemistry 276(51): 48588-48595.

Clària J, Serhan CN (1995). Aspirin triggers previously undescribed bioactive eicosanoids by human endothelial cell-leukocyte interactions. Proceedings of the National Academy of Sciences of the United States of America 92: 9475-9479.

Coffelt SB, Marini FC, Watson K, Zwezdaryk KJ, Dembinski JL, LaMarca HL (2009). The pro-inflammatory peptide LL-37 promotes ovarian tumor progression through recruitment of multipotent mesenchymal stromal cells. Proceedings of the National Academy of Sciences of the United States of America 106(10): 3806-3811.

Cross MJ, Claesson-Welsh L (2013). FGF and VEGF function in angiogenesis: signalling pathways,biological responses and therapeutic inhibition. RENDS in Pharmacological Sciences 22(4): 201-207.

Cryer B, Bhatt DL, Lanza FL, Dong JF, Lichtenberger LM, Marathi UK, et al. (2011). Low-dose aspirininduced ulceration is attenuated by aspirin-phosphatidylcholine: a randomized clinical trial. Am J Gastroenterol 106(2): 272-277.

Dai X, Yan J, Fu X, Pan Q, Sun D, Xu Y, et al. (2017). Aspirin inhibits cancer metastasis and angiogenesis via targeting heparanase. Clinical Cancer Research 23(20): 6267-6278.

Desborough MJR, Keeling DM (2017). The aspirin story-from willow to wonder drug. British Journal of Haematology 5: 674-683.

Diasio RB, Harris BE (1989). Clinical Pharmacology of 5-Fluorouracil. Clinical Pharmacokinetics 16(4): 215-237.

Eilken HM, Adams RH (2010). Dynamics of endothelial cell behavior in sprouting angiogenesis. Current Opinion in Cell Biology 22: 617-625.

Elamin YY, Rafee S, Osman N, O Byrne KJ, Gately K (2016). Thymidine Phosphorylase in Cancer; Enemy or Friend? Cancer Microenviron 9( 1): 33-43. 
Esch F, Baird A, Ling N, Ueno N, Hill F, Denoroy L, et al. (1985). Primary structure of bovine pituitary basic fibroblast growth factor (FGF) and comparison with the amino-terminal sequence of bovine brain acidic FGF. Proceedings of the National Academy of Sciences of the United States of America 82(19): 6507-11.

Etulain J, Fondevila C, Negrotto S, Schattner M (2013). Platelet-mediated angiogenesis is independent of VEGF and fully inhibited by aspirin. British journal of pharmacology 2: 255-265.

Fernández IS, Cuevas P, Angulo J, López-Navajas P, Canales-Mayordomo A, González-Corrochano R, et al. (2010). Gentisic acid, a compound associated with plant defense and a metabolite of aspirin, heads a new class of in vivo fibroblast growth factor inhibitors. The journal of biological chemistry 285(15): 11714-29.

Fierro IM, Kutok JL, Serhan CN (2002). Novel lipid mediator regulators of endothelial cell proliferation and migration: aspirin-triggered-15R-lipoxin A(4) and lipoxin A(4). The Journal of pharmacology and experimental therapeutics 300(2): 385-392.

Finetti F, Solito R, Morbidelli L, Giachetti A, Ziche M, Donnini S (2008). Prostaglandin E2 regulates angiogenesis via activation of fibroblast growth factor receptor-1. The Journal of biological chemistry 283(4): 2139-2146.

Finnis C, Dodsworth N, Pollitt CE, Carr G, Sleep D (1993). Thymidine phosphorylase activity of plateletderived endothelial cell growth factor is responsible for endothelial cell mitogenicity. European journal of biochemistry 212: 201-210.

Folkman J (1971). Tumor angiogenesis: therapeutic implications. The New England Journal of Medicine 285(21): 1182-1186.

Fox SB, Moghaddam A, Westwood M, Turley H, Bicknell R, Gatter KC, et al. (1995). Platelet-derived endothelial cell growth factor/thymidine phosphorylase expression in normal tissues: an immunohistochemical study. The Journal of pathology 176(2): 183-190.

Ghezzo F, Cesano L, Mognetti B, Pesce E, Pirro E, Corvetti G, et al. (2005). Salicylate inhibition of rat mammary carcinogenesis and angiogenesis in female rat compatible with misoprostol administration. International journal of oncology 26(3): 697-702.

Goel S, Duda DG, Xu L, Munn LL, Boucher Y, Fukumura D, et al. (2011). Normalization of the vasculature for treatment of cancer and other diseases. Physiological reviews 91: 1071-1121.

Gordon S, Martinez FO (2010). Alternative activation of macrophages: mechanism and functions. Immunity 32(5):593-604.

Goveia J, Stapor P, Carmeliet P (2014). Principles of targeting endothelial cell metabolism to treat angiogenesis and endothelial cell dysfunction in disease. EMBO molecular medicine 9: 1105-1120.

Griffioen AW, Molema G (2000). Angiogenesis: potentials for pharmacologic intervention in the treatment of cancer, cardiovascular diseases, and chronic inflammation. Pharmacological reviews 52: 237-268.

Grootveld M, Halliwell B (1988). 2,3-Dihydroxybenzoic acid is a product of human aspirin metabolism. Biochemical pharmacology 37(2): 271-80.

Haussen J, Koczulla R, Shaykhiev R, Herr C, Pinkenburg O, Reimer D, et al. (2008). The host defence peptide LL-37/hCAP-18 is a growth factor for lung cancer cells. Lung Cancer 59: 12-23.

Heffelfinger SC (2007). The Renin angiotensin system in the regulation of angiogenesis. Current Pharmaceutical Design 13: 1215-1229.

Hiraoka N, Allen E, Apel IJ, Gyetko MR, Weiss SJ (1998). Matrix metalloproteinases regulate neovascularization by acting as pericellular fibrinolysins. Cell 95: 365-377. 
Holmes CE, Jasielec J, Levis JE, Skelly J, Muss HB (2013). Initiation of aspirin therapy modulates angiogenic protein levels in women with breast cancer receiving tamoxifen therapy. Clinical and translational science 6(5): 386-390.

Hsieh CC, Wang CH (2018). Aspirin disrupts the crosstalk of angiogenic and inflammatory cytokines between 4T1 breast cancer cells and macrophages. Mediators of Inflammation 2018: 638-643.

Hu C, Dandapat A, Mehta JL (2007). Angiotensin II induces capillary formation from endothelial cells via the LOX-1-dependent redox-sensitive pathway. Hypertension 50: 952-957.

Hu C, Dandapat A, Sun L, Khan JA, Liu Y, Hermonat PL, et al. (2008). Regulation of TGFbeta1-mediated collagen formation by LOX-1: studies based on forced overexpression of TGFbeta1 in wild-type and lox-1 knock-out mouse cardiac fibroblasts. The Journal of biological chemistry 283(16):10226-10231.

Hu Y, Lou X, Wang R, Sun C, Liu X, Liu S, et al. (2014). Aspirin, a potential GLUT1 inhibitor in a vascular endothelial cell line. Open Medicine 14(1): 552-560.

Hua H, Zhang H, Kong Q, Wang J, Jiang Y (2019). Complex roles of the old drug aspirin in cancer chemoprevention and therapy. Medicinal research reviews 39(1): 114-145.

Huang Y, Lichtenberger LM, Taylor M, Bottsford-Miller JN, Haemmerle M, Wagner MJ, et al. (2016). Antitumor and Antiangiogenic Effects of Aspirin-PC in Ovarian Cancer. Molecular cancer therapeutics 15: 2894-2904.

Jain RK (1998). Delivery of molecular and cellular medicine to solid tumors. Journal of Controlled Release 53: $49-67$.

Jones MK, Wang H, Peskar BM, Levin E, Itani RM, Sarfeh IJ, et al (1999). Inhibition of angiogenesis by nonsteroidal anti-inflammatory drugs: Insight into mechanisms and implications for cancer growth and ulcer healing. Nature Medicine 5(12): 1418-1423.

Kapoor C, Vaidya S, Wadhwan V, Hitesh, Kaur G, Pathak A (2016). Seesaw of matrix metallo proteinases (MMPs). Journal of Cancer Research and Therapeutics 12(1): 28-35.

Khaidakov M, Mitra S, Mehta JL (2012). Adherence junction proteins in angiogenesis: modulation by aspirin and salicylic acid. Journal of Cardiovascular Medicine 13(3): 188-192.

Khaidakov M, Szwedo J, Mitra S, Ayyadevara S, Dobretsov M, Lu J, et al. (2010). Antiangiogenic and antimitotic effects of aspirin in hypoxia-reoxygenation modulation of the LOX-1-NADPH oxidase axis as a potential mechanism. Journal of cardiovascular pharmacology 56:635-641.

Khaidakov M, Szwedo J, Mitra S, Mehta JL (2011). Angiostatic effects of aspirin in hypoxia? Reoxygenation are linked to modulation of TGFb1 signaling. journal of cardiovascular pharmacology and therapeutics 16(1): 105-110.

Khokha R, Waterhouse P (1994). The role of tissue inhibitor of metalloproteinase-1 in specific aspects of cancer progression and reproduction. Journal of Neuro-Oncology 18(2): 123-127.

Larrick JW, Hirata M, Balint RF, Lee J, Zhong J, Wright SC (1995). Human CAP18: a novel antimicrobial lipopolysaccharide-binding protein. Infection and immunity 63(4): 1291-7.

Lavie CJ, Howden CW, Scheiman J, Tursi J (2017). Upper gastrointestinal toxicity associated with long-term aspirin therapy: consequences and prevention. Curr Probl Cardio 142(5): 146-164.

Lawson ND, Weinstein BM (2002). In Vivo Imaging of Embryonic Vascular Development Using Transgenic Zebrafish. Developmental Biology 248(2): 307-318.

Leibovich SJ, Polverini PJ, Shepard HM, Wiseman DM, Shively V, Nuseir N (1987). Macrophage-induced angiogenesis is mediated by tumour necrosis factor-alpha. Nature 329(15): 630-632. 
Li JP , Vlodavsky I (2009). Heparin, heparan sulfate and heparanase in inflammatory reactions. Thromb Haemost 102: 823-8.

Li WW, Li VW, Hutnik M, Chiou AS (2012). Tumor angiogenesis as a target for dietary cancer prevention. Journal of oncology 2012:879623.

LiekensS, De Clercq E, Neyts J (2001). Angiogenesis: regulators and clinical applications. Biochemical Pharmacology 61: 253-270.

Liu Z, Kobayashi K, van Dinther M, van Heiningen SH, Valdimarsdottir G, van Laar T, et al. (2009). VEGF and inhibitors of TGFbeta type-I receptor kinase synergistically promote blood-vessel formation by inducing alpha5-integrin expression. Journal of Cell Science 122: 3294-3302.

Ma J, Waxman DJ (2008). Combination of antiangiogenesis with chemotherapy for more effective cancer treatment. Mol Cancer Ther 7: 3670-3684.

Maity G, Chakraborty J, Ghosh A, Haque I, Banerjee S, Banerjee SK (2019). Aspirin suppresses tumor cell-induced angiogenesis and their incongruity. Journal of Cell Communication and Signaling volume 13: 491-502.

Meade EA, Smith WL, DeWitt DL (1993). Differential inhibition of prostaglandin endoperoxide synthase (cyclooxygenase) isozymes by aspirin and other non-steroidal anti-inflammatory drug. The Journal of biological chemistry 268(9): 6610-6614

Méric JB, Rottey S, Olaussen K, Soria JC, Khayat D, Rixe O, et al. (2006). Cyclooxygenase-2 as a target for anticancer drug development. Critical Reviews in Oncology/Hematology 59(1): 51-64.

Mitchell JA, Akarasereenont P, Thiemermann C, Flower RJ, Vane JR (1993). Selectivity of nonsteroidal antiinflammatory drugs as inhibitors of constitutive and inducible cyclooxygenase. Proceeding of the National Academy of Sciences of the United States of America 90(24):11693-7.

Mitra S, Wang X, Khaidakov M, Ding Z, Ayyadevera S, Hearnsberger E, et al. (2012). Aspirin downregulates angiotensin type 1 receptor transcription implications in capillary formation from endothelial cells. Journal of cardiovascular pharmacology 60(2): 187-192.

Miyadera K, Sumizawa T, Haraguchi M, Yoshida H, Konstanty W, Yamada Y, et al. (1995). Role of thymidine phosphorylase activity in the angiogenic effect of platelet derived endothelial cell growth factor/thymidine phosphorylase. Cancer research 55: 1687-1690.

Navone SE, Guarnaccia L, Cordiglieri C, Crisà FM, Caroli M, Locatelli M, et al. (2018). Aspirin affects tumor angiogenesis and sensitizes human glioblastoma endothelial cells to temozolomide, bevacizumab, and sunitinib, impairing vascular endothelial growth factor-related signaling. World Neurosurgery 120: 380-391.

Ott I, Kircher B, Bagowski CP, Vlecken DH, Ott EB, Will J, et al. (2009). Modulation of the Biological Properties of Aspirin by Formation of a Bioorganometallic Derivative. Angew. Chem. Int. Ed 48(6):11601163.

Ouyang N, Williams JL, Rigas B (2008). NO-donating aspirin inhibits angiogenesis by suppressing VEGF expression in HT-29human colon cancer mouse xenografts. Carcinogenesis 29 (9): 1794-1798.

Patan S (2000). Vasculogenesis and angiogenesis as mechanisms of vascular network formation, growth and remodeling. Journal of Neuro-Oncology 50 (1-2): 1-15.

Patzelt J, Langer HF (2012). Platelets in Angiogenesis. Current Vascular Pharmacology 10:570-577.

Pavón-Romero GF, Ramírez-Jiménez F, Roldán-Alvarez MA, Terán LM,Falfán-Valencia R (2017). Physiopathology and genetics in aspirin-exacerbated respiratory disease. Experimental lung research 43(8): 327-335.

Peach MJ (1977). Renin-angiotensin system: biochemistry and mechanisms of action. Physiological reviews 57(2): 313-370. 
Pearce HR, Kalia N, Bardhan KD, Brown NJ (2003). Effects of aspirin and indomethacin on endothelial cell proliferation in vitro. Journal of Gastroenterology and Hepatology 18(10): 1180-7.

Pipili-Synetos E, Papadimitriou E, Maragoudakis ME (1998). Evidence that platelets promote tube formation by endothelial cells on matrigel. British Journal of Phar macology 125: 1252-1257.

Puyraimond A, Weitzman JB, Babiole E, Menashi S (1999). Examining the relationship between the gelatinolytic balance and the invasive capacity of endothelial cells. Journal of Cell Science 112: 1283-1290

Radziwon-Balicka A, Moncada de la Rosa C, Jurasz P (2012). Platelet-associated angiogenesis regulating factors: a pharmacological perspective. Can. J. Physiol. Pharmacol 90(6):679-688.

Rauzi F, Kirkby NS, Edin ML, Whiteford J, Zeldin DC, Mitchell JA, et al. (2016). Aspirin inhibits the production of proangiogenic 15(S)-HETE by platelet cyclooxygenase-1. The FASEB Journal 30(12):42564266 .

Ribeiro AL, Okamoto OK (2015). Combined effects of pericytes in the tumor microenvironment. Stem cells international 2015: 868475 .

Rigas B (2007). The use of nitric oxide-donating nonsteroidal anti-inflammatory drugs in the chemoprevention of colorectal neoplasia. Current opinion in gastroenterology 23(1): 55-59

Rivera LB, Bergers G (2014). Targeting vascular sprouts: Manipulating metabolism could control angiogenesis. Science 344(6191): 1449-1450.

Rousseau S, Houle F, Kotanides H, Witte L, Waltenberger J, Landry J, et al (2000). Vascular endothelial growth factor (VEGF)-driven actin-based motility is mediated by VEGFR2 and requires concerted activation of stress-activated protein kinase 2(SAPK2/p38) and geldanamycin-sensitive phosphorylation of focal adhesion kinase. The Journal of biological chemistry 275(14): 10661-10672.

Rumble RH, Roberts MS, Wanwimolruk S (1981). Determination of aspirin and its major metabolites in plasma by high-performance liquid chromatography without solvent extraction. JonmaI of Chromatogmphy 225: 252-260.

Salcedo R, Zhang X, Young HA, Michael N, Wasserman K, Ma WH, et al. (2003). Angiogenic effects of prostaglandin E2 are mediated by up-regulation of CXCR4 on human microvascular endothelial cells. Blood 102(6): 1966-77.

Salvado MD, Di Gennaro A, Lindbom L, Agerberth B, Haeggström JZ (2013). Cathelicidin LL-37 induces angiogenesis via PGE2-EP3 signaling in endothelial cells, in vivo inhibition by aspirin. Arteriosclerosis, Thrombosis, and Vascular Biology 33(8): 1965-1972.

Saunders MA, Sansores-Garcia L, Gilroy DW, Wu KK (2001). Selective suppression of CCAAT/enhancerbinding protein beta binding and cyclooxygenase-2 promoter activity by sodium salicylate in quiescent human fibroblasts. The Journal of biological chemistry 276:18897-18904.

Seeger FH, Sedding D, Langheinrich AC, Haendeler J, Zeiher AM, Dimmeler S (2010). Inhibition of the p38 MAP kinase in vivo improves number and functional activity of vasculogenic cells and reduces atherosclerotic disease progression. Basic Res Cardiol 105: 389-397.

Semaan A, Munkarah AR, Arabi H, Bandyopadhyay S, Seward S, Kumar S, et al. (2011). Expression of GLUT-1 in epithelial ovarian carcinoma: Correlation with tumor cell proliferation, angiogenesis, survival and ability to predict optimal cytoreduction. Gynecologic Oncology 121(1): 181-186.

Shi C, Zhang N, Feng Y, Cao J, Chen X, Liu B (2017). Aspirin inhibits IKK- $\beta$-mediated prostate cancer cell invasion by targeting matrix metalloproteinase-9 and urokinase-type plasminogen activator. Cell Physiol Biochem 41: 1313-1324. 
Shtivelband MI, Juneja HS, Lee S, Wu KK (2003). Aspirin and salicylate inhibit colon cancer medium-and VEGF-induced endothelial tube formation: correlation with suppression of cyclooxygenase-2 expression. Journal of Thrombosis and Haemostasis 1(10):2225-2233.

Skultetyova D, Filipova S, Riecansky I, Skultety J (2007). The Role of angiotensin type 1 receptor in inflammation and endothelial dysfunction. Recent Patents on Cardiovascular Drug Discovery 2(1): 23-27.

Solinas G, Germano G, Mantovani A, Allavena P (2009). Tumor-associated macrophages (TAM) as major players of the cancer-related inflammation. Journal of Leukocyte Biology 86: 1065-1073.

Spiller KL, Anfang RR, Spiller KJ, Ng J, Nakazawa KR, Daulton JW, et al. (2014). The role of macrophage phenotype in vascularization of tissue engineering scaffolds. Biomaterials 35(15): 4477-4488.

Spiller KL, Nassiri S, Witherel CE, Anfang RR, Ng J, Nakazawa KR, et al. (2015). Sequential delivery of immunomodulatory cytokines to facilitate the M1-to-M2 transition of macrophages and enhance vascularization of bone scaffolds. Biomaterials 37:194-207.

$\mathrm{Su}$ BB, Chen JH, Shi H, Chen QQ, Wan J, et al. (2014). Aspirin may modify tumor microenvironment via antiplatelet effect. Medical Hypotheses 83: 148-150.

Takebayashi Y, Akiyama S, Akiba S, Yamada K, Miyadera K, Sumizawa T, et al. (1996). Clinicopathologic and prognostic significance of an angiogenic factor, thymidine phosphorylase, in human colorectal carcinoma. Journal of the National Cancer Institute 88(16): 1110-1117.

Talmadge JE, Fidler IJ(2010). AACR centennial series: the biology of cancer metastasis: historical perspective. Cancer research 70(14): 5649-5669.

Thomas KA, Rios-Candelore M, Giménez-Gallego G, DiSalvo J, Bennett C, Rodkey J, et al. (1985). Pure brain-derived acidic fibroblast growth factor is a potent angiogenic vascular endothelial cell mitogen with sequence homology to interleukin 1. Proceedings of the National Academy of Sciences of the United States of America 82(19): 6409-13.

Tsujii M, Kawano S, Tsuji S. Sawaoka H, Horin M, Dubois RN (1998). Cyclooxygenase regulates angiogenesis induced by colon cancer cells. Cell 93: 705-716

Uefuji K, Ichikura T, Mochizuki H (2000). Cyclooxygenase-2 expression is related to prostaglandin biosynthesis and angiogenesis in human gastric cancer. Clinical Cancer Research 6: 135-138.

Ushio-Fukai M (2006). Redox signaling in angiogenesis: Role of NADPH oxidase. Cardiovascular Research 71(2): $226-235$.

Vasudev NS, Reynolds AR (2014). Anti-angiogenic therapy for cancer: current progress, unresolved questions and future directions. Angiogenesis17(3): 471-494.

Vaupel P (2004). The Role of Hypoxia-Induced Factors in Tumor Progression. The Oncologist 9(5): 10-17.

Vlodavsky I, Friedmann Y (2001). Molecular properties and involvement of heparanase in cancer metastasis and angiogenesis. The Journal of Clinical Investigation 108: 341-347.

Vlodavsky I, Gross-Cohen M, Weissmann M, Ilan N, Sanderson RD (2018). Opposing functions of heparanase-1 and heparanase-2 in cancer progression. Trends in Biochemical Sciences43(1): 18-31.

Weber G, Chamorro CI, Granath F, Liljegren A, Zreika S, Saidak Z, et al. (2009). Human antimicrobial protein hCAP18/LL-37 promotes a metastatic phenotype in breast cancer. Breast Cancer Research 11(1): R6.

Weis SM, Cheresh DA (2011). Tumor angiogenesis: molecular pathways and therapeutic targets. Nature Medicine 17(11): 1359-1370. 
Worathumrong N, Grimes AJ (1975). Anaerobic glycolysis in normal human erythrocytes incubated in vitro with sodium salicylate. Clinical Science and Molecular Medicine 49: 375-384.

Xu XM, Sansores-Garcia L, Chen XM, Matijevic-Aleksic N, Du M, Wu KK. (1999). Suppression of inducible cyclooxygenase 2 gene transcription by aspirin and sodium salicylate. Proceedings of the National Academy of Sciences of the United States of America 96(9):5292-5297.

Yang YY, Hu CJ, Chang SM, Tai TY, Leu SJ (2004). Aspirin inhibits monocyte chemoattractant protein-1 and interleukin-8 expression in TNF-astimulated human umbilicaln vein endothelial cells. Atherosclerosis 174: $207-213$.

Yeh RK, Chen J, Williams JL, Baluch M, Hundley TR, Rosenbaum RE, et al. (2004). NO-donating nonsteroidal antiinflammatory drugs (NSAIDs) inhibit colon cancer cell growth more potently than traditional NSAIDs: a general pharmacological property? Biochemical Pharmacology 67: 2197-2205.

Yoshida S, Amano H, Hayashi I, Kitasato H, Kamata M, Inukain M, et al. (2003). COX-2/VEGF-dependent facilitation of tumor-associated angiogenesis and tumor growth in vivo. Laboratory investigation; a journal of technical ethods andpathology 83(10): 1385-94.

Zapata-Morales JR, Galicia-Cruz OG, Franco M, Martinez Y, Morales F (2014). Hypoxia-inducible factor- $1 \alpha$ $($ HIF-1 $\alpha$ ) protein diminishes sodium glucose transport 1 (SGLT1) and SGLT2 protein expression in renal epithelial tubular cells (LLC-PK1) under hypoxia. The Journal of biological chemistry 289(1): 346-357.

Zaslavsky A, Baek KH, Lynch RC, Short S, Grillo J, Folkman J, et al. (2010). Platelet-derived thrombospondin-1 is a critical negative regulator and potential biomarker of angiogenesis. Blood 115(22):4605-4613.

Zhang B, Cao H, Rao GN (2005). 15(S)-Hydroxyeicosatetraenoic acid induces angiogenesis via activation of PI3K-Akt-mTOR-S6K1 signaling. Cancer Res 65(16): 7283-7291.

Zhang X, Wang Z, Wang Z, Zhang Y, Jia Q, Wu L, et al. (2013). Impact of acetylsalicylic acid on tumor angiogenesis and lymphangiogenesis through inhibition of VEGF signaling in a murine sarcoma model. Oncology reports 29(5): 1907-1913.

Zhu GH, Schwartz EL (2003). Expression of the angiogenic factor thymidine phosphorylase in THP-1 monocytes: induction by autocrine tumor necrosis factor-alpha and inhibition by aspirin. Molecular pharmacology 64(5): 1251-1258. 